\title{
Effect of Functional Fitness on Plasma Oxidation Level in Elders: Reduction of the Plasma Oxidants and Improvement of the Antioxidant Barrier
}

\author{
Tania Gamberi ${ }^{1}$, Giulia Gorini ${ }^{1}$, Tania Fiaschi ${ }^{2}$, Gabriele Morucci $^{2}$, Simone Pratesi ${ }^{2}$, \\ Lucio Fittipaldi $^{1}$, Massimo Gulisano ${ }^{2}$, Pietro Amedeo Modesti ${ }^{2}$, Alessandra Modesti ${ }^{1}$, \\ Francesca Magherini ${ }^{1, *}$ \\ ${ }^{1}$ Department of Biomedical, Experimental and Clinical Sciences "Mario Serio", University of Florence, Florence, Italy \\ ${ }^{2}$ Department of Experimental and Clinical Medicine, University of Florence, Florence, Italy
}

Email address:

tania.gamberi@unifi.it(T.Gamberi), giulia.gorini@unifi.it(G. Gorini), tania.fiaschi@unifi.it(T.Fiaschi), gabri.morucci@gmail.com(G. Morucci), simonepratesi@unifi.it(S.Pratesi), luciofittipaldi@gmail.com(L.Fittipaldi), massimo.gulisano@unifi.it(M.Gulisano),pamodesti@unifi.it(P.A. Modesti), alessandra.modesti@unifi.it(A. Modesti), francesca.magherini@unifi.it(F.Magherini)

${ }^{*}$ Corresponding author

\section{To cite this article:}

Tania Gamberi, Giulia Gorini, Tania Fiaschi, Gabriele Morucci, Simone Pratesi, Lucio Fittipaldi, Massimo Gulisano, Pietro Amedeo Modesti, Alessandra Modesti, Francesca Magherini. Effect of Functional Fitness on Plasma Oxidation Level in Elders: Reduction of the Plasma Oxidants and Improvement of the Antioxidant Barrier. American Journal of Sports Science. Vol. 6, No. 2, 2018 , pp. 55-64. doi: 10.11648/j.ajss.20180602.14

Received: December 19, 2017; Accepted: February 24, 2018; Published: April 9, 2018

\begin{abstract}
Aging is characterized by a progressive decline in the physiological function due to the gradual alteration of molecules, cells and tissues. Reactive oxygen species (ROS) are the by-product of aerobic metabolism, and their increase is physiologically counteracted by the activation of the antioxidant machinery. A typical hallmark of aging is the imbalance of such equilibrium, due to either an increase of the amount of radicals or a failure of the antioxidant system. Literature reports that physical exercise is able to restore and maintain the homeostasis of oxidants and antioxidants during aging. Recently, growing interest has been turned to functional fitness, a special physical activity aimed to enhance the ability to perform everyday tasks, such as dressing, climbing stairs and preparing meals. The aim of this work was to assess whether a 24 weeksfunctional fitness program carried out on 28 elderly participants (57-86 years old) could be able to improve their oxidative status. For this purpose, dROMs (diacron Reactive Oxygen Metabolites) and BAP (Biological Antioxidant Potential) were analysed at the beginning and at the end of the study. Furthermore, both plasma and saliva protein carbonylation levels were explored through proteomics analysis.
\end{abstract}

Keywords: Aging, Oxidative Stress, Functional Fitness, Protein Carbonylation

\section{Introduction}

Aging is characterized by several alterations in molecules, cells and tissues that lead to a progressive decline of the physiological function and loss of resistance [1]. A typical modification occurring in aging is the gradual imbalance of the oxidative stress: the Free Radical Theory of Aging first developed and described in 1956 [2] states that aging is characterized by a high production of reactive oxygen species
(ROS). Under normal cellular conditions, ROS are produced as result of aerobic metabolism and exposure to endogenous and exogenous toxic agents. Physiological ROS concentrations are important for cell signaling and homeostasis and excess or free radicals is promptly counteracted by the scavenging activity of the antioxidant system [3]. During aging, such balance is compromised due 
to either an increase of ROS or a decrease in the efficiency of the antioxidant machinery, or both of these [4]. This higher level of ROS may damage all types of biological molecules including proteins and lipids in plasma of older people. Among these targets, proteins are the most vulnerable to oxidative stress since ROS are able to directly bind amino acids residues and generate stable carbonyl moieties [3]. For this reason, protein carbonyls are used as common marker for identification and quantification of protein damage caused by oxidative stress [5]. Moreover, a significant failure in plasma antioxidant capacity during aging has been described in respect of a decreased reducing ability of plasma towards oxidized thiol groups [6].

It has been observed that regular physical exercise is essential for healthy aging and beneficial for cardiovascular system [7] and cognitive function [8]. With regard to oxidative stress, physical activity is able to regulate both proand anti-oxidant activities by preventing the exacerbation of free radical release related to older age. This event contributes to postponing the aging process and decreasing the incidence of diseases associated with oxidative stress [9].

In a previous paper, a characterization of plasma proteins that undergo oxidation in response to physical exercise in young athletes was performed. By proteomic analysis, a class of proteins was found oxidized after physical exercise and, on the contrary, several proteins showed a reduced oxidation after the physical activity. Serotransferrin and Fibrinogen, the most susceptible proteins to oxidation, showed a reduced carbonylation level after physical activity confirming that their reduced level of oxidation was due to a beneficial effect of physical exercise [5].

On the other hand, an important feature of physical exercise is the increase of serum cortisol levels [10]. Cortisol is defined as the stress hormone, since it is secreted by the hypothalamic-pituitary-adrenal axis in response to stressing stimuli, such as physical activity. There is evidence suggesting that physical exercise promotes an increase of cortisol levels in both younger and older people, but throughout the aging process, the adaptation and recovery from stress are less efficient, and this can lead to a higher susceptibility to older age diseases. However, the entity of stress is strongly influenced by both the type and the intensity of physical exercise [11].

Healthy aging needs to be characterized by the capacity of maintaining adequate levels of physical function not only to control the oxidative status, but also to carry out basic tasks of everyday life, such as dressing, eating, climbing stairs independently, as well as shopping, walking and preparing meals without undue fatigue [12]. To handle with these demands, a special type of training program has been developed. It is called functional fitness and aims to maintain and improve the physical independence of older adults in their daily life by enhancing cardiac, respiratory and muscular joint functions (e.g. flexibility and balance). For this purpose, a functional fitness training program normally includes postural, stretching and aerobic exercises [13].
Taking all these aspects into account, the aim of this work was to shed light on the importance of functional fitness in regulating the oxidative stress of elderly adults. To clarify this, 28 volunteers aged 57-86 were recruited and administered a 24 weeks-functional fitness program aimed to facilitate and improve basic daily tasks. The training program was set in order to be moderate and adequate for the age of the participants. An analysis of the modulation of plasma and saliva oxidative stress was carried out, along with the evaluation of salivary cortisol levels and of the main anthropometric parameters.

\section{Materials and Methods}

\subsection{Participants}

To realize the present study, twenty-eight healthy older adults ( 21 women and 7 men) were recruited. All participants volunteered to participate following explanation of all experimental procedures and signed an informed consent form prior to the enrolment in the study. Besides, The Institutional Review Board at the University of Florence, Italy, approved this project.

Volunteers were aged 57-86 and not engaged in any regular exercise in the last year. The inclusion criteria were: old adults, aged $>55$, who were all capable of performing basic daily activities and living on their own. The exclusion criteria were cognitive impairment and mental disability, as well as smoking and drinking habits, overweight, obesity or diabetes. In addition, none of the participants used to take antioxidant supplements. Participants were recruited at Palestra Olimpia, Impianto Sportivo Polivalente "Danilo Innocenti", Sesto F.no, Florence, Italy, where training sessions took place with the permission of the members of the center.

All participants had to undergo a medical examination that revealed no contraindications to physical exercise. They were asked to provide information regarding prescribed medications. Participants under hormone replacement treatment, corticosteroids and antiresorptive therapies were excluded. In addition, to limit the effects of possible variables on the results, medicines and their doses were not altered throughout the months.

All measurements necessary for the realization of the present study, as well as plasma and saliva samples, were taken both at the beginning (T0) and at the end of the study after 24 weeks (T1).

\subsection{Functional Fitness Training Description}

Participants completed a 24 weeks-training program including stretching, flexibility, balance and postural exercises with two sessions per week on non-consecutive days. Sessions took place in specific rooms with peaceful and pleasant environment. Sessions lasted for 45-50 minutes and were led by the same exercise trainer who maintained all the settings throughout the months. The sessions were divided into three main parts: warm-up (5-10 minutes, including 
postural and stretching exercises for general activation), specific training (35 minutes including moderate aerobic exercises and muscular tasks for coordination/balance training) and cool-down (5 minutes with respiratory and flexibility exercises).

\subsection{Body-Composition Measurements}

Weight and height of all participants were measured on a calibrated balance. Afterwards, BMI (body mass index) was calculated as the ratio between the weight $(\mathrm{kg})$ and the squared height $\left(\mathrm{m}^{2}\right)$, and finally expressed as $\mathrm{kg} / \mathrm{m}^{2}$.

\subsection{Plasma Oxidative Stress Analysis}

A fasting capillary blood sample was taken from each volunteer both at $\mathrm{T} 0$ and $\mathrm{T} 1$ by using a heparinized microvette. Plasma was extracted through centrifugation (6000 rpm for 90 seconds) of the entire blood sample (about $300 \mu \mathrm{L})$ and stored at $4^{\circ} \mathrm{C}$ until oxidative stress measurements. During all handling procedures including centrifugation, the temperature was maintained at $4{ }^{\circ} \mathrm{C}$. All analyses were performed immediately after the blood collection. We evaluated the plasma levels of reactive oxygen metabolites and the antioxidant capacity using the d-ROMs (diacron reactive oxygen metabolites) test and the BAP (Biological Antioxidant Potential) test, used to determine the efficiency of the plasma barrier to balance the production of free radicals in terms of iron-reducing activity. The biomarkers ROM and BAP were selected based on their short- and long-term stability. All plasma analyses were performed by using a free radical analyzer system (FREE Carpe Diem, DIACRON INTERNATIONAL s.r.l) that included a spectrophotometric device reader and a thermostatically regulated mini-centrifuge, and the measurement kits were optimized to the FREE Carpe Diem System, according to the manufacturer's instructions. The dROMs test reflects the amount of organic hydroperoxides that is related to the free radicals from which they are formed. When samples are dissolved in an acidic buffer, proteins release metals ions $(\mathrm{Fe})$. The hydroperoxides react with these and are converted to alkoxy and peroxy radicals. These newly formed radicals oxidize an additive aromatic amine $(\mathrm{N}, \mathrm{N}-$ diethylparaphenylen-diamine) and cause the formation of a relatively stable colored cation radical that is spectrophotometrically detectable at $505 \mathrm{~nm}$ [14]. The results are expressed in arbitrary units (Carr U.), one unit of which corresponds to $0.8 \mathrm{mg} / \mathrm{L}$ of hydrogen peroxide [15]. The BAP test provides an estimation of the global antioxidant capacity of blood plasma, measured as its reducing potential against ferric ions. The intensity of the decoloration resulting by mixing a ferric chloride solution with a thiocyanate derivative solution is spectrophotometrically detectable at $505 \mathrm{~nm}$ and is proportional to the ability of plasma to reduce ferric ions. The results are expressed in $\mu \mathrm{mol} / \mathrm{L}$ of the reduced ferric ions [16]

\subsection{Salivary Cortisol Assay}

Saliva samples were taken from all participants, as described in Pacini et al., 2014 [17], at T0 and T1 by using the Salivette ${ }^{\circledR}$ device (Sarsted, Verona, Italy). In particular, participants were instructed to circulate the swab in the mouth for 2 minutes until it was filled with saliva. Once saliva had been collected from all participants, cortisol concentration was assayed through a competitive immuneenzymatic colorimetric method for quantitative determination (DiaMetra, Milano, Italy): cortisol present in the samples competes with cortisol conjugated to horseradish peroxidase (HRP) for the antibody binding sites on a microtiter plate. After incubation, unbounds components are washed away and the remaining bound cortisol is measured through the reaction of $\mathrm{H}_{2} \mathrm{O}_{2}$ with tetramethylbenzidine (TMB) catalyzed by HRP. The reaction produces a blue color. Then, the addition of an acidic solution stops the reaction and produces a yellow color, whose intensity is inversely proportional to the amount of cortisol present in the sample. Salivary cortisol concentration was expressed as $\mathrm{ng} / \mathrm{ml}$.

\subsection{Protein Carbonylation Analysis}

Plasma and saliva samples were collected as described above and pooled separately in two groups ( $\mathrm{T} 0$ and $\mathrm{T} 1)$ to proceed with the experiment. Albumin and $\operatorname{IgG}$ were depleted from T0 and T1 plasma samples as reported in Guidi et al., 2011 [5]. Saliva samples were treated with trichloroacetic acid (TCA) to precipitate proteins, as described in the TCA protein precipitation protocol (originally from Luis Sanchez, revised October 2001). Both plasma and saliva extracts from T0 and T1 groups were separately suspended in $8 \mathrm{M}$ urea, 4\% 3-[(3Cholamidopropyl) dimethylammonio]-1-propanesulfonate (CHAPS), $65 \mathrm{mM}$ dithiothreitol (DTT). Proteins were quantified through Bradford colorimetric method (Biorad, Hercules, California, USA) and $80 \mu \mathrm{g}$ were separated on 11 $\mathrm{cm}$ immobilized $\mathrm{pH}$ gradient (IPG) strips ( $\mathrm{pH} 3-10 \mathrm{NL}$ ) on a PROTEAN i12 IEF System (BIO-RAD) as described in Carpentieri et al, 2016 [18]. Next, the strips were incubated in $2 \mathrm{~N} \mathrm{HCl}$ with $10 \mathrm{mM} \mathrm{2,4-dinitrophenyl-hydrazine,}$ $\mathrm{DNPH}$ (Sigma, St. Louis, MO) for $20 \mathrm{~min}$ at $25^{\circ} \mathrm{C}$, to enable the derivatization of protein-bound carbonyls as reported in Carpentieri et al. 2016 [18]. Once ready, strips were loaded on a second dimension $9-16 \%$ polyacrylamide gel to perform SDS-PAGE. Afterwards, proteins were blotted onto a PVDF membrane and incubated with the anti-DNP IgG antibody overnight at $4^{\circ} \mathrm{C}$. Following the incubation with the secondary antibody, the PVDF membrane was treated with ECL kit (Immobilon Western Chemiluminescent AP substrate, Millipore) to detect protein bands. Following the experiment, PVDF membranes were stained in Coomassie-blue to reveal the total amount of proteins necessary for the normalization. Images acquisition and quantification were carried out by using ImageMaster 2D Platinum software 6.0 (GE Healthcare) as previously described [5]: volumes occupied by all spots were taken and normalized to the volumes occupied by Coomassie-stained spots and compared. Statistical analysis 
was performed by calculating the mean and the standard deviation of the mean (SDM) of three experimental replicates and significance was calculated by Student's t test $(* \mathrm{p}<0.05$ and $* * \mathrm{p}<0.01)$.

\subsection{Statistical Analysis}

The statistical analysis not regarding the carbonylation assay was performed by using GraphPad Prism 6 software. For each parameter, all values taken at T0 (initial point) and T1 (final point) were averaged and the standard deviation of the mean (SDM) was calculated. The results were presented as mean \pm SDM and $95 \%$ confidence intervals (CI). Statistical significance of the means was evaluated with Student's t test: when results were statistically significant $\mathrm{P}$ value was $<0.05(* \mathrm{p}<0.05$; ** $\mathrm{p}<0.01)$.

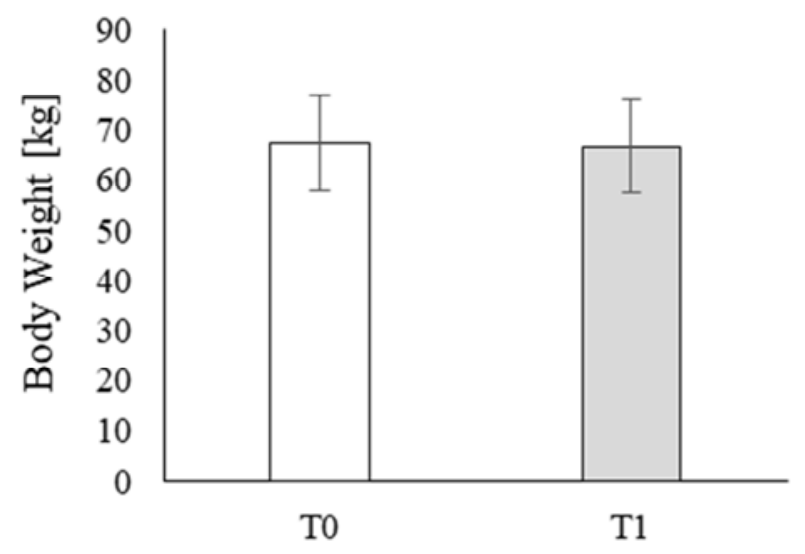

\section{Results}

\subsection{Assessment of the Anthropometric Parameters}

All measures were performed both at the beginning (T0) and after 24 weeks (T1). The intervention included 28 participants predominantly females (21 women and 7 men) and was started by examining the anthropometric parameters. Among the general characteristics, no significant differences were found. The average age of all participants was $71.96 \pm 8.15$ years and their mean height was $162 \pm 8 \mathrm{~cm}$. At $\mathrm{T} 0$, the average weight of all participants was $66.72 \pm 9.37 \mathrm{~kg}$ and the mean of BMI was $25.69 \pm 2.05 \mathrm{~kg} / \mathrm{m}^{2}$. Since according to the World Health Organization (WHO) BMI values may be different depending on age [19], the obtained measurements lay within the normal ranges of BMI for the elderly adults [20]. At T1, no significant differences were found with respect to the initial values [Figure 1].

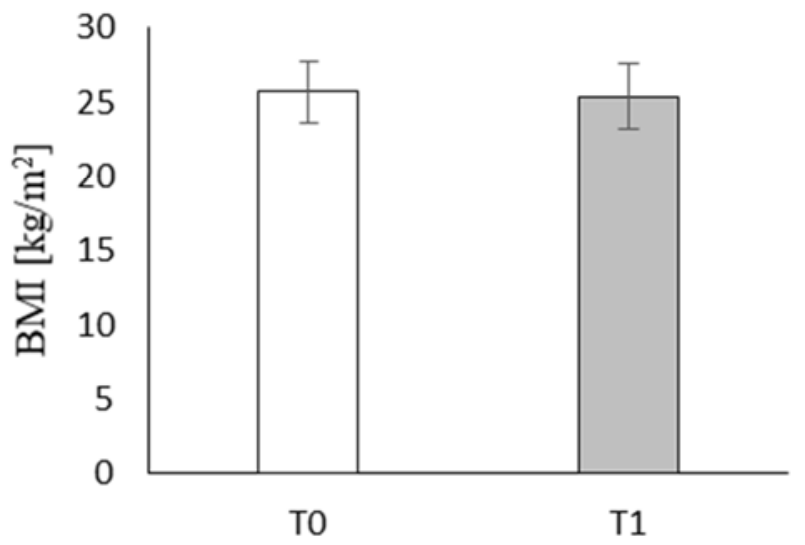

Figure 1. Evaluation of weight and BMI in all participants at T0 and T1. Results are reported as mean of weight (kg) and BMI (kg/m $\left.{ }^{2}\right)$.

\subsection{Effect of Functional Fitness on Salivary Cortisol Levels}

It is well known that cortisol is secreted in response to stressing physical exercise [10]. Hence, it was decided to assess saliva cortisol level at $\mathrm{T} 0$ and $\mathrm{T} 1$ with regard to functional fitness in order to measure the stress level in response to the proposed physical exercise. At T0, salivary cortisol concentration was found $4.18 \pm 2.35 \mathrm{ng} / \mathrm{ml}$. According to literature [21], T0 cortisol levels lay within the normal range. At $\mathrm{T} 1$, cortisol level did not change significantly $(4.99 \pm 3.41 \mathrm{ng} / \mathrm{ml})$ [Table 1]. This result can be explained by the fact that the training program was meant to be moderate and adequate for $\sim 70$ years old participants.

Table 1. Effect of 24 weeks-functional fitness on 28 elderly participants with regard to anthropometric parameters (weight and BMI), plasma cortisol concentration, plasma oxidative stress and antioxidant potential. [SDM=standard deviation of the mean, 95\% C.I. =95\% confidence interval, $p=p$ value obtained by Student's $t$ test; $T 0=$ initial point, $T 1=$ final point $]$.

\begin{tabular}{llll}
\hline & Mean \pm SDM & Mean \pm SDM & 95\% C. I. \\
\hline & T0 & T1 & \\
\hline Weight $[\mathrm{kg}]$ & $67.52 \pm 9.48$ & $66.72 \pm 9.37$ & -7.002 to 5.402 \\
BMI $[\mathrm{kg} / \mathrm{m} 2]$ & $25.69 \pm 2.05$ & $25.39 \pm 2.24$ & -1.705 to 1.21 \\
Body fat mass [\%] & $36.96 \pm 5.61$ & $35.08 \pm 6.51$ & -5.878 to 2.120 \\
Cortisol $[\mathrm{ng} / \mathrm{ml}]$ & $4.18 \pm 2.35$ & $4.99 \pm 3.41$ & -1.122 to 2.728 \\
dROMs $[\mathrm{u} . \mathrm{carr}]$ & $402.16 \pm 84.74$ & $346.70 \pm 59.42$ & -103.6 to -7.304 \\
$\mathrm{H}_{2} \mathrm{O}_{2}[\mathrm{mg} / \mathrm{dl}]$ & $32.17 \pm 6.78$ & $27.3 \pm 4.75$ & 0,347 \\
BAP $[\mu \mathrm{mol} / \mathrm{l}]$ & $1575.79 \pm 307.94$ & $2091.94 \pm 142.03$ & 0,4034 \\
\hline
\end{tabular}




\subsection{Plasma Oxidative Stress Evaluation}

$\boldsymbol{A}$

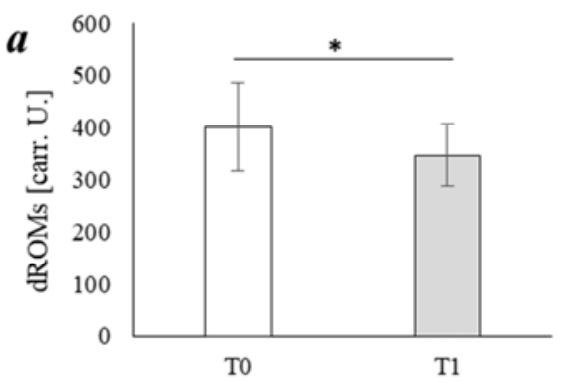

B

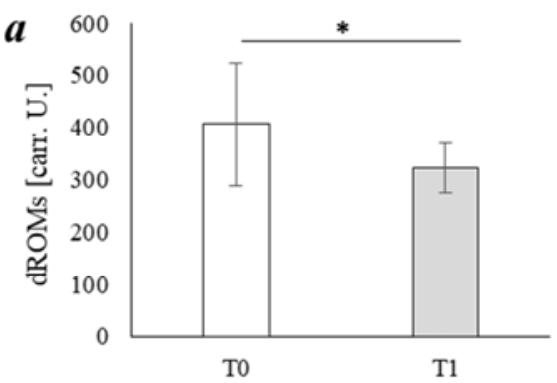

C

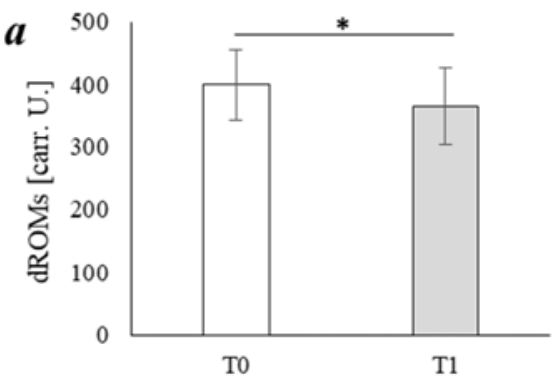

D

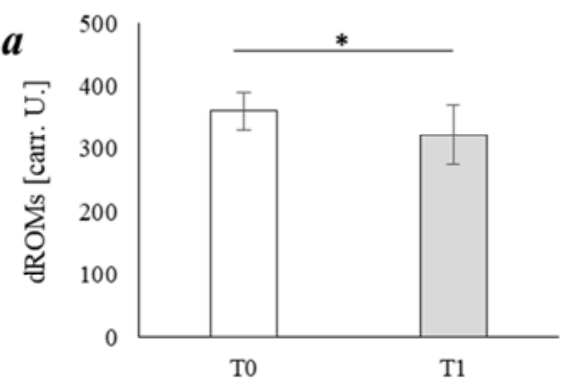

$\boldsymbol{E}$

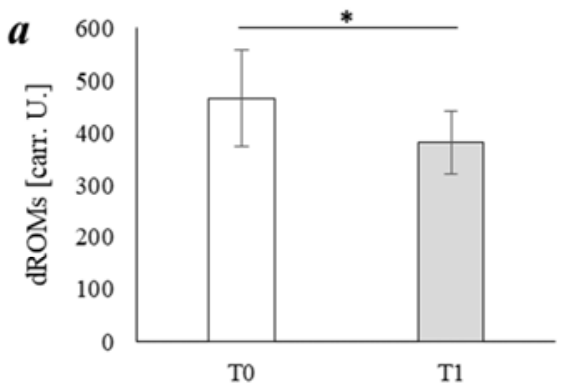

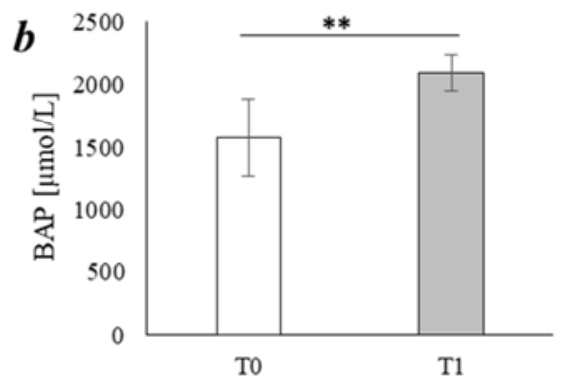
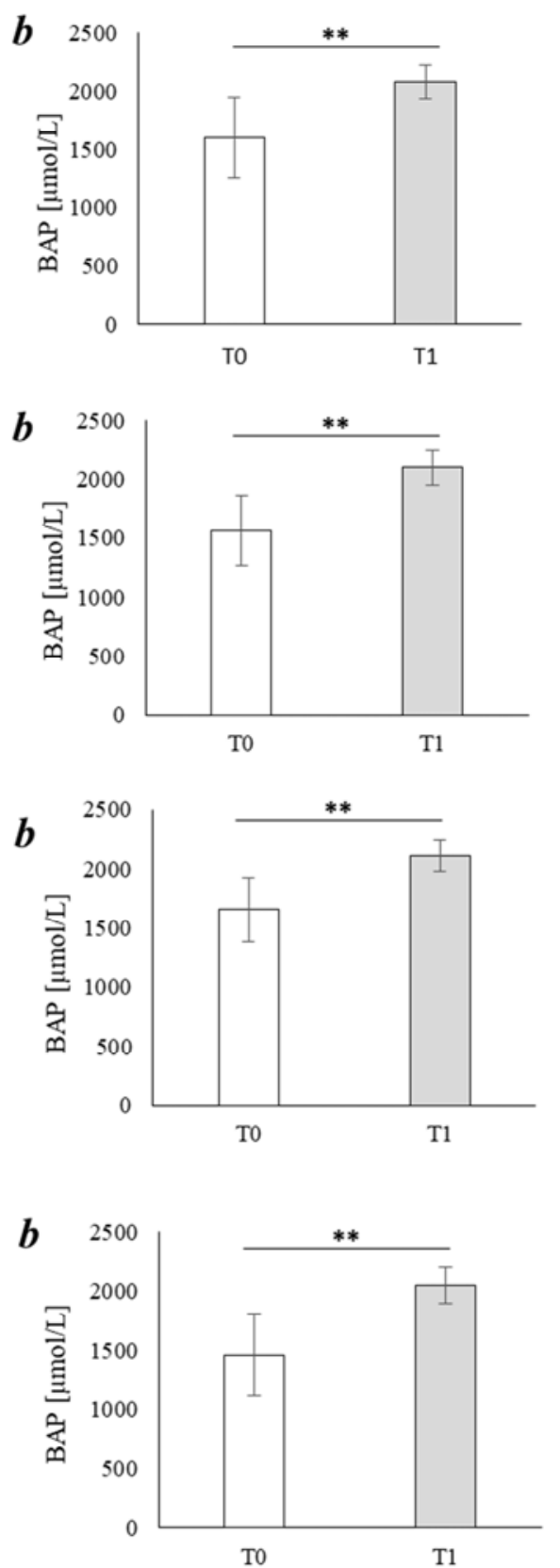

Figure 2. Plasma dROMs (left) and BAP (right) levels participants at time T0 (white bars) and T1 (grey bars).

Panel A reports the evaluation in all participants, Panel B in middle-aged participants and Panel C in older participants.

Panel D represents the values obtained in participants starting from low/middle level of oxidative stress.

Panel E shows the values in participants with a high/very high initial level of oxidative stress. Results are expressed as carr. u. (Carratelli units) for dROMs and as $\mu \mathrm{mol} / \mathrm{L}$ for BAP. ${ }^{*} \mathrm{p}<0.05 ; * * \mathrm{p}<0.01$. 
To evaluate the effects of 24 weeks-functional fitness program on the oxidative status of the participants, plasma levels of oxidative and antioxidative parameters were respectively measured at $\mathrm{T} 0$ and $\mathrm{T} 1$. First, an analysis of the average level of both dROMs and BAP in all participants at the beginning of the program was performed. The dROMs average value observed at T0 was $402.17 \pm 84.74$ carr.u. According to literature [15], ROMs values can be classified depending on the severity of the oxidative stress: 250-300 carr.u.: normal level of oxidative stress; 300-400 carr.u.: low/middle level of oxidative stress; $>400$ carr.u.: high/very high level of oxidative stress. Therefore, initial dROMs values of participants lay within the higher level of oxidative stress, and this may be due to their older age. As it is shown in the graphs of Figure $2 A$, a , at T1 a significant decrease of dROMs of $14 \%$ (from $402.17 \pm 84.74$ carr.u to $346.71 \pm 59.42$ carr.u) was observed. Along with this, BAP average level was $1575.80 \pm 307.94 \mu \mathrm{mol} / \mathrm{L}$ at $\mathrm{T} 0$. It has been reported that BAP levels above $2200 \mu \mathrm{mol} / \mathrm{L}$ are considered within the normal range of biological antioxidant potential [22]. Hence, the initial BAP level observed in all participants indicated a high deficiency status, probably related to their older age. As shown in Figure 2A, b, BAP average value significantly increased of $33 \%$ at $\mathrm{T} 1$ (from $1575.80 \pm 307.94 \mu \mathrm{mol} / \mathrm{L}$ to $2091.95 \pm 142.03 \mu \mathrm{mol} / \mathrm{L}$ ). Therefore, the proposed functional fitness program actually reduced the amount of plasma oxidants and improved the biological antioxidant potential bringing dROMs nearer to the normal ranges. Moreover, from dROMs values $\mathrm{H}_{2} \mathrm{O}_{2}$ concentration was calculated $(\mathrm{mg} / \mathrm{dL})$ and it was found significantly decreased $(-14 \%)$ at T1 [Table 1]. The second step was to assess whether dROMs decrease might be different depending on age. For this purpose, the whole group of participants was divided into two smaller groups: the first group included 13 middleaged participants (57-70 years old), and the second one included 15 older participants (aged 71-86) [Figure 2, panels $B$ and $C$, respectively]. For both groups the average values of dROMs [Figure $2 B, a$ and $2 C, a$ ] and BAP [Figure $2 B, b$ and $2 C, b$ ] were calculated. Initial dROMs values were found very similar between the two groups $(406.13 \pm 118.31$ carr.u. for the first group and $399.29 \pm 55.75$ carr.u. for the second group), indicating that dROMs initial values were not affected by the age of the participants. As shown in the figure, dROMs significantly decreased in both cases. Interestingly, the decrease was of $21 \%$ for the first group (from $406.13 \pm 118.31$ carr.u. at T0 to $321.63 \pm 47.78$ carr.u. at T1) and of $9 \%$ for the second one (from $399.29 \pm 55.75$ carr.u. at T0 to $364.95 \pm 62.36$ carr.u. at T1). This result suggests that middle-aged participants gave a higher contribution to the decrease of dROMs previously described in panel A. Likewise, $\mathrm{H}_{2} \mathrm{O}_{2}$ concentration decreased in both groups (data not shown). Furthermore, the evaluation of BAP showed that T0 values were similar between the groups $(1594.96 \pm 346.29 \mu \mathrm{mol} / \mathrm{L}$ for the first group and $1561 \pm 293.66 \mu \mathrm{mol} / \mathrm{L}$ for the second group). As reported in the graphs, a significant increase in both cases was found (+30-35\%), regardless of the age of the participants. Indeed, BAP values at $\mathrm{T} 1$ were found $2084.39 \pm 144.75 \mu \mathrm{mol} / \mathrm{L}$ in the first group and $2097.45 \pm 146.85 \mu \mathrm{mol} / \mathrm{L}$ in the second group.

A further analysis of the same set of data was carried out considering the indications given by the manufacturer and found in literature [15]. Since none of the participants' initial dROMs started from normal levels of oxidative stress, 320 carr.u. were considered the threshold value for our analysis. All participants were divided into two groups according to their initial values of dROMs. The first group included 16 participants starting from low/middle level of oxidative stress (320-400 carr.u.), whereas the second one included 12 participants starting from high/very high level of oxidative stress (400-680 carr.u.) [Figure 2, panels $D$ and $E$ respectively]. Both dROMs and BAP changes were analyzed. In the first group dROMs levels significantly decreased of $10 \%$ (from $360.20 \pm 30.34$ carr.u. to $322.50 \pm 47.61$ carr.u.) [Figure 2D, a] and BAP levels significantly increased of $28 \%$ (from $1653.44 \pm 272.39 \mu \mathrm{mol} / \mathrm{L}$ to $2109.30 \pm 136.66$ $\mu \mathrm{mol} / \mathrm{L})[$ Figure $2 D, b]$. Interestingly, in the second group, a higher and significant decrease of dROMs $(-18 \%)$ and a higher and significant increase of BAP $(+40 \%)$ were observed. In particular, dROMs initial levels at T0 were $466.65 \pm 93.24$ carr.u. and became $382.18 \pm 60.35$ carr.u. at T1 [Figure 2E, a]. BAP values at T0 were $1463.36 \pm 351.02$ $\mu \mathrm{mol} / \mathrm{L}$ and became $2052.94 \pm 151.49 \mu \mathrm{mol} / \mathrm{L}$ at $\mathrm{T} 1$ [Figure $2 E, b]$. Noteworthy, one participant showed an initial value of 680 carr.u. which decreased of $\sim 40 \%$ at $\mathrm{T} 1$ (data not shown).

All these results suggest that the 24-weeks functional fitness program induced a significant improvement in the oxidative status of elderly adults. In particular, middle-age participants showed a higher decrease of plasma oxidants levels. Moreover, the worse was the initial oxidative status of participants, the higher was the improvement on both plasma oxidant and antioxidants levels.

\subsection{Plasma and Saliva Protein Carbonylation}

In order to confirm the results obtained from the analysis of dROMs, all plasma samples were pooled separately in $\mathrm{T} 0$ and $\mathrm{T} 1$, and both dROMs and BAP of the pooled samples were analyzed. Once again, a decrease of dROMs $(-10 \%)$ and an increase of BAP $(+30 \%)$ were observed (not shown).

These results prompted us to investigate the level of protein carbonylation on $\mathrm{T} 0$ and $\mathrm{T} 1$ pools of both plasma and saliva. Comparison and normalization of protein carbonyls were carried out by matching the 2D-oxyblots and the 2D-Coomassie stained PVDF membranes coming from the samples. Figure 3 shows images of 2D-oxyblots of T0 and T1 pooled plasma [panels $A$ and $B$ ] and saliva [panels $C$ and $D$ ]. Images are representative of three experimental replicates carried out on the same samples. To evaluate the effect of functional fitness on protein carbonylation, the volumes occupied by all spots of both T0 and T1 2D-oxyblots were normalized to the volumes 
occupied by the spots detected in their respective Coomassie-stained PVDF membranes [Figure 4]. The analysis showed that the volume of spots in T0 plasma was 97.9 \pm 12.5 A.U. and became $72.4 \pm 10$ A.U. at T1. The graph reported in Figure 3, a, shows that the training program significantly reduced plasma protein carbonylation of about
$26 \%$, thus confirming the decrease observed through dROMs analysis performed on plasma of all participants. In addition, the 2D-oxyblots of saliva carbonylation showed that at T0 the volume occupied by the spots was $99.7 \pm 15$ A.U. and became $42.3 \pm 18$ A.U. at T1. Specifically, the decrease was significant and of about 55\% (Figure3, $b$ ).
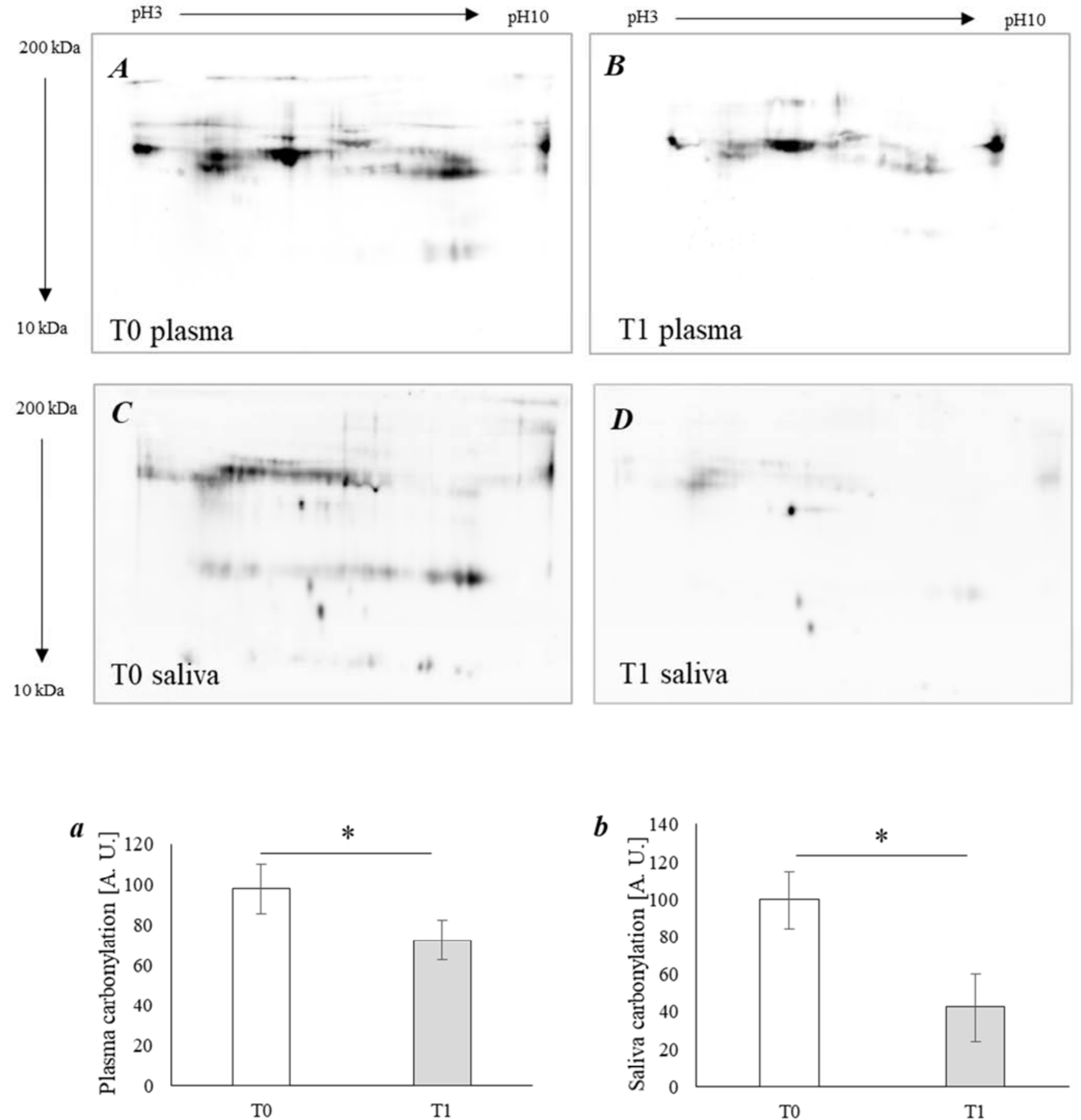

Figure 3. Representative images of the oxyblots of plasma (panels A and B) and saliva (panels, C and D) obtained from T0 (left) and T1 (right) pools.

The carbonylation level has been normalized to the corresponding Coomassie-stained PVDF membranes (see in Figure 4) and is reported in graph $a$ for plasma and graph $b$ for saliva, at T0 (white bars) and T1 (grey bars). Results are expressed as Arbitrary Units (A.U.) corresponding to the volumes occupied by the spots normalized to the volumes of total loaded protein. * $p<0.05$ 

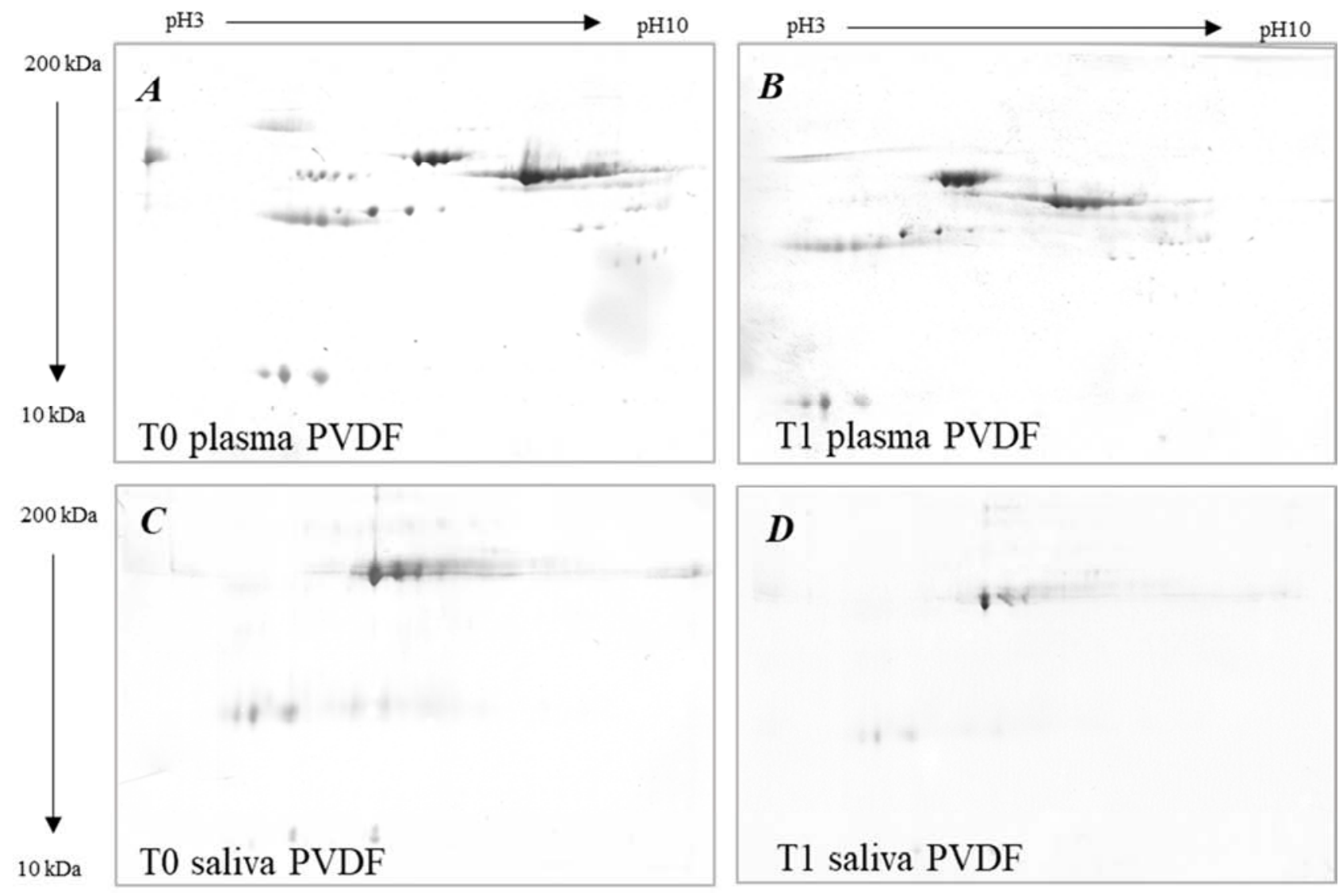

Figure 4. Representative images of the Coomassie-stained PVDF membranes of plasma (upper panels, $A$ and B) and saliva (bottom panels, C and D) obtained from $T 0$ and T1 pools. Left panels correspond to T0 pools and right panels represent T1 pools.

\section{Discussion}

The 24 weeks-functional fitness program proposed in the present study did not cause any alterations of weight and BMI of the participants. In fact, it was not meant to induce neither a weight of loss nor a reduction of BMI since the initial values of both weight and BMI lay within normal ranges in all participants, according to the World Health Organization (WHO). In addition, although we did not find significantly lower values of BMI in most of elderly people practicing regular functional fitness, all participants increased the adaptability to oxidative stress. During aging, the body becomes more susceptible to oxidation so that intense physical activity may increase cellular ROS production and induce a state of cellular damage leading to oxidative stress. Our results show that the proposed functional fitness program improved the redox status of our sample of 28 elderly adults by reducing the reactive oxygen metabolites of $20 \%$ and enhancing the plasma antioxidant potential of about $30 \%$. Rowinski et al [23] observed an increased activity of the antioxidant enzyme SOD in elders as a consequence of different types of physical activity. Other authors analyzed different markers of the antioxidant capacity and demonstrated a positive correlation between the concentration of these markers and the intensity of the physical exercise, even though the different types of physical activity reported in that study were determined through subjective questionnaires [24].

Our results support the hypothesis that regular physical activity and particularly an activity associated with various health benefits like the functional fitness program proposed in the present study, has beneficial effects on the balance between plasma ROS production and the ability to neutralize them.

One of the limitations of our study is certainly the small sample size. To overcome this matter, the fitness program was strictly thought and organized in order to be administered and followed by a specific personal trainer who maintained all the settings throughout the period of observation. The final purpose was ameliorating both physical health and the independence of the participants in performing every-day tasks.

Furthermore, the results obtained from the analysis of the biological antioxidant potential were confirmed by plasma and saliva protein carbonylation measurements through 2DGE technique, which showed a significant decrease of protein carbonylation in both plasma and saliva in response to the proposed functional fitness program. The reduction of protein carbonylation level may be explained by a proteasome-dependent degradation, as reported in a previous publication ${ }^{5}$, although it has been discovered that a regulated decarbonylation process defines transient kinetics of carbonylation signals in certain conditions [25].

Handling the oxidative stress during aging is an important 
goal, since it has been seen that one of the conditions underlying sarcopenia, a physiological weakening of skeletal muscles occurring in aging, is an enhancement of oxidants in plasma and muscular tissues [26]. Therefore, the positive effects of such physical training relate to an achievement of independence during aging, improvement of muscular strength and function given by exercise and maintenance of the redox homeostasis.

\section{Conclusions}

The most important observation coming from the present study is that functional fitness decreases oxidative stress in elderly adults, in terms of reduction of plasma and saliva oxidants, such as organic hydroperoxides and protein carbonyls, and enhances the efficiency of the antioxidant barrier.

Secondly, neither weight nor BMI are modified by the proposed functional training, and salivary cortisol levels do not change significantly, indicating that this type of physical activity is moderate and appropriate for older adults.

Taken together, all the data obtained suggest that functional fitness may be introduced as an ordinary daily training, both to preserve independence of elders and to control their oxidative status.

\section{References}

[1] da Cruz AC, Petronilho F, Heluany CC, Vuolo F, Miguel SP, Quevedo J, Romano-Silva MA, Dal-Pizzol F., 'Oxidative stress and aging: correlation with clinical parameters', Aging Clin. Exp. Res., vol. 26, no. 1, pp. 7-12, Feb. 2014.

[2] D. Harman, 'Aging: a theory based on free radical and radiation chemistry', J. Gerontol., vol. 11, no. 3, pp. 298-300, Jul. 1956.

[3] K. B. Pandey, M. M. Mehdi, P. K. Maurya, and S. I. Rizvi, 'Plasma protein oxidation and its correlation with antioxidant potential during human aging', Dis. Markers, vol. 29, no. 1, pp. 31-36, 2010.

[4] D. Harman, 'The free radical theory of aging', Antioxid. Redox Signal., vol. 5, no. 5, pp. 557-561, Oct. 2003.

[5] Guidi F, Magherini F, Gamberi T, Bini L, Puglia M, Marzocchini R, Ranaldi F, Modesti PA, Gulisano M, Modesti A., 'Plasma protein carbonylation and physical exercise', Mol. Biosyst., vol. 7, no. 3, pp. 640-650, Mar. 2011.

[6] S. I. Rizvi, R. Jha, and P. K. Maurya, 'Erythrocyte plasma membrane redox system in human aging', Rejuvenation Res., vol. 9, no. 4, pp. 470-474, 2006.

[7] A. M. Jacomini, D. D. Dias, J. O. Brito, R. F. da Silva, H. L. Monteiro, S. Llesuy, K. De Angelis, S. L. Amaral, A. S. Zago, 'Influence of Estimated Training Status on Anti and Pro-Oxidant Activity, Nitrite Concentration, and Blood Pressure in Middle-Aged and Older Women', Front. Physiol., vol. 8, Mar. 2017.

[8] M. Berchicci, G. Lucci, and F. Di Russo, 'Benefits of Physical Exercise on the Aging Brain: The Role of the Prefrontal
Cortex', J. Gerontol. A. Biol. Sci. Med. Sci., vol. 68, no. 11, pp. 1337-1341, Nov. 2013.

[9] Cebula A, Tyka AK, Pilch W, Szyguła Z, Pałka T, SztafaCabała K, Frączek B, Tyka A, 'Effects of 6-week Nordic walking training on body composition and antioxidant status for women $>55$ years of age', Int. J. Occup. Med. Environ. Health, vol. 30, no. 3, pp. 445-454, May 2017.

[10] E. E. Hill, E. Zack, C. Battaglini, M. Viru, A. Viru, and A. C. Hackney, 'Exercise and circulating cortisol levels: the intensity threshold effect', J. Endocrinol. Invest., vol. 31, no. 7, pp. 587-591, Jul. 2008.

[11] D I Corazza, E Sebastião, R Valle Pedroso, C A Almeida Andreatto, F Gomes de Melo Coelho, S Gobbi, E Teodorov, R Ferreira Santos-Galduróz, 'Influence of chronic exercise on serum cortisol levels in older adults', Eur. Rev. Aging Phys. Act., vol. 11, no. 1, pp. 25-34, Apr. 2014.

[12] L. L. de Melo, V. H. Menec, and A. E. Ready, 'Relationship of functional fitness with daily steps in community-dwelling older adults', J. Geriatr. Phys. Ther. 2001, vol. 37, no. 3, pp. 116-120, Sep. 2014.

[13] Yang HJ1, Chen KM, Chen MD, Wu HC, Chang WJ, Wang YC, Huang HT, 'Applying the transtheoretical model to promote functional fitness of community older adults participating in elastic band exercises', J. Adv. Nurs., vol. 71, no. 10 , pp. 2338-2349, Oct. 2015.

[14] U. Cornelli, R. Terranova, S. Luca, M. Cornelli, and A. Alberti, 'Bioavailability and antioxidant activity of some food supplements in men and women using the D-Roms test as a marker of oxidative stress', J. Nutr., vol. 131, no. 12, pp. 3208-3211, Dec. 2001.

[15] R. Trotti, M. Carratelli, and M. Barbieri, 'Performance and clinical application of a new, fast method for the detection of hydroperoxides in serum', Panminerva Med., vol. 44, no. 1, pp. 37-40, Mar. 2002.

[16] Martinovic J, Dopsaj V, Dopsaj MJ, Kotur-Stevuljevic J, Vujovic A, Stefanovic A, Nesic G., 'Long-term effects of oxidative stress in volleyball players', Int. J. Sports Med., vol. 30, no. 12, pp. 851-856, Dec. 2009.

[17] Pacini S., Branca J. J., Gulisano M., Levi Micheli M., Ceroti M., Ruggiero M., Morucci G., 'Salivary testosterone and cortisol levels to assess conditioning training program in rugby union players', Med. Sport (Roma), vol. 67, pp. 449463, Sep. 2014.

[18] Carpentieri A, Gamberi T, Modesti A, Amoresano A, Colombini B, Nocella M, Bagni MA, Fiaschi T, Barolo L, Gulisano M, Magherini F, 'Profiling Carbonylated Proteins in Heart and Skeletal Muscle Mitochondria from Trained and Untrained Mice', J. Proteome Res., vol. 15, no. 10, pp. 36663678, Oct. 2016.

[19] 'WHO | Obesity: preventing and managing the global epidemic', WHO. [Online]. Available: http://www.who.int/entity/nutrition/publications/obesity/WHO _TRS_894/en/index.html. [Accessed: 26-Sep-2017].

[20] 'Body mass index: MedlinePlus Medical Encyclopedia'. [Online]. Available: https://medlineplus.gov/ency/article/007196.htm. [Accessed: 25-Sep-2017]. 
[21] G. H. Ice, A. Katz-Stein, J. Himes, and R. L. Kane, 'Diurnal cycles of salivary cortisol in older adults', Psychoneuroendocrinology, vol. 29, no. 3, pp. 355-370, Apr. 2004.

[22] Martarelli D, Verdenelli MC, Scuri S, Cocchioni M, Silvi S, Cecchini C, Pompei $\mathrm{P}$ 'Effect of a probiotic intake on oxidant and antioxidant parameters in plasma of athletes during intense exercise training', Curr. Microbiol., vol. 62, no. 6, pp. 1689-1696, Jun. 2011.

[23] R. Rowiński, M. Kozakiewicz, K. Kędziora-Kornatowska, E. Hübner-Woźniak, and J. Kędziora, 'Markers of oxidative stress and erythrocyte antioxidant enzyme activity in older men and women with differing physical activity', Exp. Gerontol., vol. 48, no. 11, pp. 1141-1146, Nov. 2013.
[24] Fraile-Bermúdez AB, Kortajarena M, Zarrazquin I, Maquibar A, Yanguas JJ, Sánchez-Fernández CE, Gil J, Irazusta A, Ruiz-Litago F, 'Relationship between physical activity and markers of oxidative stress in independent community-living elderly individuals', Exp. Gerontol., vol. 70, pp. 26-31, Oct. 2015.

[25] C. M. Wong, L. Marcocci, L. Liu, and Y. J. Suzuki, 'Cell signaling by protein carbonylation and decarbonylation', Antioxid. Redox Signal., vol. 12, no. 3, pp. 393-404, Mar. 2010.

[26] B. Can. O. Kara, M. C. Kizilarslanoglu, G. Arik, G. S. Aycicek, F. Sumer, R. Civelek, C. Demirtas, Z. Ulger, 'Serum markers of inflammation and oxidative stress in sarcopenia', Aging Clin. Exp. Res., vol. 29, no. 4, pp. 745-752, Aug. 2017. 